Pure and Applied Mathematics Quarterly

Volume 8, Number 3

$589-608,2012$

\title{
Complex Dynamics in A Financial Model ${ }^{*}$
}

\author{
Yinghui Gao, Wei Feng and Bing Liu
}

\begin{abstract}
A discrete two-dimensional financial model is investigated. The conditions of existence for flip bifurcation and Naimark-Sacker bifurcation are derived using center manifold theorem and bifurcation theory. Chaotic behavior in the sense of Marotto's definition of chaos is proven. And numerical simulations not only show the consistence with the theoretical analysis but also exhibit the complex dynamical behaviors, including quasi-period orbits, interior crisis and intermittency. The computation of Lyapunov exponents conforms the dynamical behaviors.
\end{abstract}

Keywords: discrete two-dimensional financial model, bifurcation, Marotto chaos.

\section{Introduction}

In $[1,15]$, the authors developed a discrete time model of asset price dynamics containing the essential elements of the heterogenous interacting agents paradigm. The model reduces to a two-dimensional nonlinear map. The authors analyzed the global dynamic behaviors in detail using the method of critical curves and they also indicated that the map undergoes flip and Naimark-Sacker bifurcations. But they did not theoretically prove the existence of those two kinds of bifurcations. Here, we will do it and prove that attracting invariant closed curve bifurcates from the Naimark-Sacker bifurcation point under certain conditions. In [12],

Received July 9, 2008

* This work is supported by National Natural Science Foundation of China under Grant Nos. 10971009, 10971001 and by the Fundamental Research Funds for the Central Universities. 
a fixed point $p^{*}$ is called a snap-back repeller of $f$ if: $(\mathrm{j} 1) p^{*}$ is a fixed point of $f$ with all eigenvalues of $D f\left(p^{*}\right)$ exceeding 1 in magnitude; $(\mathrm{j} 2)$ there exists a point $x_{0} \neq p^{*}$ in a repelling neighborhood of $p^{*}$, such that $x_{M}=p^{*}$ and $\operatorname{det}\left(D f\left(x_{k}\right)\right) \neq 0$ for $0 \leq k<M$, where $x_{k}=f^{k}\left(x_{0}\right)$. Repelling neighborhood means that for any $x$ in such a neighborhood $B_{r}\left(p^{*}\right)$, the pre-image points $f^{-k}(x)$ remain within the local unstable manifold for all $k \geq 0$ (although not necessarily within $B_{r}\left(p^{*}\right)$ ), and $f^{-k}(x) \rightarrow p^{*}$ as $k \rightarrow \infty$. But Gardini ${ }^{[3]}$ indicated that the condition $(\mathrm{j} 2)$ can be generalized to $(\mathrm{j} 2)^{\prime}$ : there exists a point $p$ homoclinic to $p^{*}$, $p \in U\left(p^{*}\right)$, and the homoclinic orbit associated with $p, \mathcal{O} p$ is noncritical, where $U\left(p^{*}\right)$ is a neighborhood of $p^{*}$ such that all the eigenvalues of $D f(x)$ exceeding 1 in magnitude, $\forall x \in U\left(p^{*}\right)$ and $f\left(U\left(p^{*}\right)\right) \supset U\left(p^{*}\right)$. The former is easy to use in proving the existence of Marotto chaos for varying parameters. So, here, we investigate chaos using the theorem in [12]. A related study in [2] is also concerned with the existence of Marotto chaos in a very similar model. But the authors only did it for a specific set of parameters. In this paper, we will prove that there exists Marotto chaos for varying parameters under certain conditions.

As we have seen, many nonlinear systems have parameters which appear in the defining systems of equations. As the parameter is changed, changes may occur in the qualitative structure of the orbits for certain parameter values ${ }^{[4]}$. A central problem in nonlinear dynamics is that of discovering how the properties of orbits change and evolve as a parameter of a dynamical system is changed $^{[14]}$. So, we mainly discuss the changing properties of the map. Among them, bifurcations and chaos are very important phenomena which many excellent researchers $[6,7,8,16,17]$ apply themselves to study.

Here, we firstly study the stability of the fixed points, then analyze the bifurcations of the system using center manifold theorem and bifurcation theory $[4,18]$. We find that there exist flip bifurcation and Naimark-Sacker bifurcation.

In $1975, \mathrm{Li}$ and Yorke ${ }^{[9]}$ proved that period three implies chaos on the interval. From then on, many mathematicians began to devote their research to further exploring the complex mechanics of the nonlinear transformations, especially in high-dimensional systems. Among them, the result, given by Marotto ${ }^{[11]}$, on the criterion for chaos existence in high-dimensional systems is, of course standout and surprising. A snap-back repeller, just like period three in nonlinear trans- 
formations, is regarded as an inducement to produce chaos in high-dimensional systems. Thus, this criterion is widely used in proving the existence of chaos in many nonlinear dynamical systems ${ }^{[10]}$. Many years after this work first appeared, it was brought to Marotto's attention that there is a minor technical flaw in the reasoning he used in some of his arguments. A fixed point $z$ is referred to as repelling under $f$ if all eigenvalues of $D f(z)$ exceed 1 in magnitude. But $z$ is expanding only if $\|f(x)-f(y)\|>s\|x-y\|$, where $s>1$, for all $x, y$ sufficiently close to $z$ with $x \neq y$. Although all expanding fixed points are repelling, the converse is not true. As a result, Marotto's original definition of a snap-back repeller and proof that the existence of such a point implies chaos are in error. But Marotto quickly realized that the flaw is of a minor technical nature. According to the discussion by M. Hirsch and S. Smale in [5], this eigenvalue condition does imply the existence of a vector in $R^{n}$ for which the above inequality is true. However, during the past decade or so several papers have appeared that first overstate the severity of the error, and then propose correct but profoundly altered and weakened versions of his theorem. Marotto believed they were mistaken and gave a better version of Marotto theorem ${ }^{[12]}$. Here, we rigorously prove that the discrete two-dimensional financial model possesses chaotic phenomenon using the better version.

This paper is organized as follows. In section 2, we describe first the model and the meaning of the parameters. In section 3 , we show that there exist flip bifurcation and Naimark-Sacker bifurcation using center manifold theorem and bifurcation theory. It is rigorously proven that the two-dimensional discrete nonlinear map possesses a snap-back repeller in section 4 . The results of numerical simulations and the computations of Lyapunov exponents are presented in section 5 to verify the theoretical analysis and display the complex and interesting dynamics.

\section{The model.}

What in this section is quoted from [1] and [15].

The price of the asset traded in the market depends on the interaction of two groups of agents, chartists and fundamentalists. Let us denote by $z_{t} \operatorname{logarithm}$ of the asset price at time $t$. The price evolves according to:

$$
z_{t+1}=z_{t}+\beta\left[d_{t}^{(f)}+d_{t}^{(c)}\right]
$$


where $d_{t}^{(f)}$ and $d_{t}^{(c)}$ are the fundamentalists' and chartists' demand at time $t$, respectively, and $\beta(\beta>0)$ measures the price reaction to the excess demand $D_{t}=d_{t}^{(f)}+d_{t}^{(c)}$. The fundamentalists' demand is given by $d_{t}^{(f)}=a\left(W-z_{t}\right), a>$ 0 , where $W$ is the rationally expected fundamental value of the asset. The chartists' demand is given by $d_{t}^{(c)}=\alpha \arctan \left(x_{t}-g\right), \alpha>0$, where $x_{t}=E_{t}\left[z_{t+1}\right]-$ $z_{t}$ is the chartists' expectation at time $t$ of the log-price change between $t$ and $t+1$, while $g$ is the return on an alternative asset. Finally, chartists' expectations evolve according to the following adaptive scheme:

$$
x_{t+1}=x_{t}+c\left[\left(z_{t+1}-z_{t}\right)-x_{t}\right],
$$

where $c, 0<c<1$, has the meaning of speed of adjustment of expectations to past price changes. Equations $(*)$ and $(* *)$ define a nonlinear two-dimensional system in the dynamic variables $x_{t}, z_{t}$, which has the unique steady state:

$$
\bar{x}, \bar{z}=\left(0, W+\frac{\alpha \arctan (-g)}{a}\right) .
$$

By introducing the price deviation $y_{t}=z_{t}-\bar{z}$, the system is reduced to the iteration of the following two-dimensional map:

$$
\left\{\begin{array}{l}
x \mapsto(1-c) x-c \beta[a y-k(x)] \\
y \mapsto y-\beta[a y-k(x)]
\end{array}\right.
$$

where $k(x)=\alpha[\arctan (x-g)-\arctan (-g)], a>0, \alpha>0, \beta>0,0 \leq c \leq 1$, and having the origin $O=(0,0)$ as unique fixed point.

\section{Bifurcations.}

Now we consider the discrete model (1).

Let $F(x, y)=((1-c) x-c \beta[a y-k(x)], y-\beta[a y-k(x)])$, then

and

$$
D F(x, y)=\left(\begin{array}{cc}
1-c+\frac{\alpha \beta c}{1+(x-g)^{2}} & -a \beta c \\
\frac{\alpha \beta}{1+(x-g)^{2}} & 1-a \beta
\end{array}\right)
$$

then

$$
D F(0,0)=\left(\begin{array}{cc}
1-c+\frac{\alpha \beta c}{1+g^{2}} & -a \beta c \\
\frac{\alpha \beta}{1+g^{2}} & 1-a \beta
\end{array}\right),
$$

$$
\begin{aligned}
& \operatorname{tr}(D F(0,0))=2-a \beta-c+\frac{\alpha \beta c}{1+g^{2}} \triangleq t r_{0}, \\
& \operatorname{det}(D F(0,0))=1-a \beta-c+a \beta c+\frac{\alpha \beta c}{1+g^{2}} \triangleq d_{0} .
\end{aligned}
$$

A fixed point $\bar{x}$ is (locally) asymptotically stable if the eigenvalues $\kappa_{1}$ and $\kappa_{2}$ 
of the Jacobian matrix $A(\bar{x})$, calculated at the fixed point, are less than one in modulus. The necessary and sufficient conditions guaranteeing that $\left|\kappa_{1}\right|<1$ and $\left|\kappa_{2}\right|<1$ are $^{[13]}$

$$
\begin{aligned}
& (1) 1+\operatorname{tr}(A(\bar{x}))+\operatorname{det}(A(\bar{x}))>0, \\
& (2) 1-\operatorname{tr}(A(\bar{x}))+\operatorname{det}(A(\bar{x}))>0, \\
& (3) 1-\operatorname{det}(A(\bar{x}))>0 .
\end{aligned}
$$

Here,

$1-t r_{0}+d_{0}=a \beta c>0$,

$1+t r_{0}+d_{0}=4-2 a \beta-2 c+a \beta c+\frac{2 \alpha \beta c}{1+g^{2}}$

$1-d_{0}=a \beta+c-a \beta c-\frac{\alpha \beta c}{1+g^{2}}$.

Through simple calculation, when $c>\frac{2(a \beta-2)}{a \beta-2+\frac{2 \alpha \beta}{1+g^{2}}} \triangleq c_{0}$, we have $1+t r_{0}+d_{0}>$ 0 and when $c<\frac{a \beta}{a \beta-1+\frac{\alpha \beta}{1+g^{2}}} \triangleq c_{1}$, we have $1-d_{0}>0$. Under the condition $4 \alpha \beta-\left(1+g^{2}\right)(a \beta-2)^{2}>0$, we have $c_{0}<c_{1}$. So, when $4 \alpha \beta-\left(1+g^{2}\right)(a \beta-2)^{2}>0$ and $c_{0}<c<c_{1}$ the origin is stable.

In analysis of bifurcations $c$ is as bifurcation parameter.

Theorem 1. If $a \beta>2,8+3 g-24 g^{2}+3 g^{3}<0$ and $4 \alpha \beta-\left(1+g^{2}\right)(a \beta-2)^{2}>0$, then $\alpha_{1}>0, \alpha_{2}>0$. Hence, the map (1) undergoes a flip bifurcation at $(0,0)$ for $c=c_{0}$, and the period-2 points that bifurcate from this fixed point are stable.

The proof of Theorem 1 is in the Appendix I.

We next consider the Naimark-Sacker bifurcation of map (1).

The characteristic equation associated with the linearization of map (1) about the fixed point $(0,0)$ is given by

$$
\lambda^{2}+p(c) \lambda+q(c)=0
$$

Where

$$
p(c)=-t r_{0}=-2+a \beta+c-\frac{\alpha \beta c}{1+g^{2}}, q(c)=d_{0}=1-a \beta-c+a \beta c+\frac{\alpha \beta c}{1+g^{2}} .
$$

For $c=c_{1}$, the eigenvalues of the characteristic equation are

$$
\begin{aligned}
\lambda, \bar{\lambda} & =\left.\frac{(2-a \beta-c)\left(1+g^{2}\right)+\alpha \beta c \pm \sqrt{c^{2}\left(1+g^{2}-\alpha \beta\right)^{2}-2 a \beta\left(1+g^{2}\right)\left(1+g^{2}+\alpha \beta\right) c+a^{2} \beta^{2}\left(1+g^{2}\right)^{2}}}{2\left(1+g^{2}\right)}\right|_{c=c_{1}} \\
& =\frac{2 \alpha \beta-\left(1+g^{2}\right)\left(a^{2} \beta^{2}-2 a \beta+2\right) \pm i a \beta \sqrt{\left(1+g^{2}\right)\left[4 \alpha \beta-\left(1+g^{2}\right)(a \beta-2)^{2}\right]}}{2\left[\left(1+g^{2}\right)(a \beta-1)+\alpha \beta\right]}
\end{aligned}
$$

for $4 \alpha \beta-\left(1+g^{2}\right)(a \beta-2)^{2}>0$ and they are complex conjugate with modulus 1 .

And $d=\left.\frac{d|\lambda(c)|}{d c}\right|_{c=c_{1}}=\frac{\left(1+g^{2}\right)(a \beta-1)+\alpha \beta}{2\left(1+g^{2}\right)}>0$ for $a \beta>1$. In addition, $2 \alpha \beta-$ 
$\left(1+g^{2}\right)\left(a^{2} \beta^{2}-2 a \beta+2\right)>0$ leads to $\lambda^{n}\left(c_{1}\right) \neq 1, n=1,2,3,4$.

Through complex calculations, a coefficient $\mathbf{a}$ is obtained(see the Appendix II).

Theorem 2. The map (1) undergoes a Naimark-Sacker bifurcation at the fixed point $(0,0)$ for $c=c_{1}$ if $a \beta>1,4 \alpha \beta-\left(1+g^{2}\right)(a \beta-2)^{2}>0,2 \alpha \beta-(1+$ $\left.g^{2}\right)\left(a^{2} \beta^{2}-2 a \beta+2\right)>0$ and $\mathbf{a} \neq 0$ in (7). Moreover, if $\mathbf{a}<0($ resp. $\mathbf{a}>0)$, then an attracting(resp. repelling) invariant closed curve bifurcates from the fixed point for $c>c_{1}$ (resp. $\left.c<c_{1}\right)$.

\section{Existence of Marotto chaos.}

In this section, we rigorously prove that map (1) possesses chaotic behavior in the sense of Marotto's definition.

We first present Marotto chaos definition and theorem which are quoted from [11] and [12].

Definition 1. ${ }^{[12]}$. Suppose $z$ is a fixed point of $f$ with all eigenvalues of $D f(z)$ exceeding 1 in magnitude and suppose there exists a point $x_{0} \neq z$ in a repelling neighborhood $B_{r}(z)$ of $z$ such that $x_{M}=z$ and $\operatorname{det}\left(D f\left(x_{k}\right)\right) \neq 0$ for $0 \leq k<M$, where $x_{k}=f^{k}\left(x_{0}\right)$. Then $z$ is called a snap - back repeller of $f$. Repelling neighborhood means that for any $x$ in such a $B_{r}(z)$, the pre-image points $f^{-k}(x)$ remain within the local unstable manifold for all $k \geq 0$ (although not necessarily within $B_{r}(z)$ ), and $f^{-k}(x) \rightarrow z$ as $k \rightarrow \infty$.

Marotto Theorem ${ }^{[\mathbf{1 1}]}$. If $f$ possesses a snap-back repeller, then the map $f$ is chaotic in the sense of Marotto. That is, there exist

(i) a positive integer $N$ such that for each integer $p \geq N, f$ has a point of period $p$;

(ii) a "scrambled set" of $f$, i.e., an uncountable set $S$ containing no periodic points of $f$ such that:

(a) $f(S) \subset S$,

(b) for every $x, y \in S$ with $x \neq y$

$$
\limsup _{k \rightarrow \infty}\left\|f^{k}(x)-f^{k}(y)\right\|>0,
$$

(c) for every $x \in S$ and any periodic point $y$ of $f$

$$
\limsup _{k \rightarrow \infty}\left\|f^{k}(x)-f^{k}(y)\right\|>0
$$


(iii) an uncountable subset $S_{0}$ of $S$ such that for every $x, y \in S_{0}$ :

$$
\liminf _{k \rightarrow \infty}\left\|f^{k}(x)-f^{k}(y)\right\|=0 .
$$

Now we theoretically give the conditions of existence of chaotic phenomena for map (1) in the sense of Marotto's definition of chaos.

Assume that $\kappa_{1}, \kappa_{2}$ are the eigenvalues of $A$, then $\frac{1}{\kappa_{1}}, \frac{1}{\kappa_{2}}$ are the eigenvalues of $A^{-1}$ and the necessary and sufficient conditions guaranteeing that $\left|\kappa_{1}\right|>1$ and $\left|\kappa_{2}\right|>1\left(\right.$ i.e. $\frac{1}{\left|\kappa_{1}\right|}<1$ and $\left.\frac{1}{\left|\kappa_{2}\right|}<1\right)$ are:

$$
\begin{aligned}
& (1) 1+\operatorname{tr} A^{-1}+\operatorname{det} A^{-1}>0, \\
& (2) 1-\operatorname{tr} A^{-1}+\operatorname{det} A^{-1}>0, \\
& (3) 1-\operatorname{det} A^{-1}>0 .
\end{aligned}
$$

Then

$$
\begin{aligned}
& 1+\operatorname{tr}\left[D F(x, x)^{-1}\right]+\operatorname{det}\left[D F(x, y)^{-1}\right]=1+\frac{\operatorname{tr}[D F(x, y)]}{\operatorname{det}[D F(x, y)]}+\frac{1}{\operatorname{det}[D F(x, y)]} \\
&=\frac{1+\operatorname{tr}[D F(x, y)]+\operatorname{det}[D F(x, y)]}{\operatorname{det}[D F(x, y)]}, \\
& 1-\operatorname{tr}\left[D F(x, y)^{-1}\right]+\operatorname{det}\left[D F(x, y)^{-1}\right]=1-\frac{\operatorname{tr}[D F(x, y)]}{\operatorname{det}[D F(x, y)]}+\frac{1}{\operatorname{det}[D F(x, y)]} \\
&=\frac{1-\operatorname{tr}[D F(x, y)]+\operatorname{det}[D F(x, y)]}{\operatorname{det}[D F(x, y)]}, \\
& 1-\operatorname{det}\left[D F(x, y)^{-1}\right]=1-\frac{1}{\operatorname{det}[D F(x, y)]}=\frac{\operatorname{det}[D F(x, y)]-1}{\operatorname{det}[D F(x, y)]} . \\
& \operatorname{tr}[D F(x, y)]=2-a \beta-c+\frac{\alpha \beta c}{1+(x-g)^{2}}, \operatorname{det}[D F(x, y)]=1-a \beta-c+a \beta c+\frac{\alpha \beta c}{1+(x-g)^{2}} \\
& \operatorname{det}[D F(x, y)]>0 \text { leads to } g-\sqrt{\frac{\alpha \beta c}{(a \beta-1)(1-c)}-1}<x<g+\sqrt{\frac{\alpha \beta c}{(a \beta-1)(1-c)}-1} \text { for } \\
& c>\frac{a \beta-1}{a \beta-1+\alpha \beta} .
\end{aligned}
$$

Under the condition $\operatorname{det}[D F(x, y)]>0$ we have

$1+\operatorname{tr}\left[D F(x, y)^{-1}\right]+\operatorname{det}\left[D F(x, y)^{-1}\right]>0$ is equivalent to $1+\operatorname{tr}[D F(x, y)]+$ $\operatorname{det}[D F(x, y)]>0$, which leads to $g-\sqrt{\frac{2 \alpha \beta c}{(a \beta-2)(2-c)}-1}<x<g+\sqrt{\frac{2 \alpha \beta c}{(a \beta-2)(2-c)}-1}$ for $c>\frac{2(a \beta-2)}{a \beta-2+\frac{2 \alpha \beta}{1+g^{2}}}=c_{0}$,

$1-\operatorname{tr}\left[D F(x, y)^{-1}\right]+\operatorname{det}\left[D F(x, y)^{-1}\right]=\frac{a \beta c}{\operatorname{det}[D F(x, y)]}>0$,

$1-\operatorname{det}\left[D F(x, y)^{-1}\right]>0$ is equivalent to $\operatorname{det}[D F(x, y)]-1>0$, which leads to $g-\sqrt{\frac{\alpha \beta c}{a \beta+c-a \beta c}-1}<x<g+\sqrt{\frac{\alpha \beta c}{a \beta+c-a \beta c}-1}$ for $c>\frac{a \beta}{a \beta-1+\alpha \beta}$.

Comparing the above inequalities, we obtain the condition guaranteeing that all eigenvalues of $D F(x, y)$ exceed 1 in magnitude is $g-\sqrt{\frac{\alpha \beta c}{a \beta+c-a \beta c}-1}<x<$ $g+\sqrt{\frac{\alpha \beta c}{a \beta+c-a \beta c}-1}$ for $c>\frac{a \beta}{a \beta-1+\alpha \beta}$. 
We require that $\sqrt{\frac{\alpha \beta c}{a \beta+c-a \beta c}-1}>g$, i.e., $c>\frac{a \beta}{a \beta-1+\frac{\alpha \beta}{1+g^{2}}}=c_{1}$. Under the condition $4 \alpha \beta-\left(1+g^{2}\right)(a \beta-2)^{2}>0$, we have $c_{0}<c_{1}$.

According to Definition 1, we now find a neighborhood $U=\left\{(x, y) \in R^{2} \mid g-\right.$ $\left.\sqrt{\frac{\alpha \beta c}{a \beta+c-a \beta c}-1}<x<g+\sqrt{\frac{\alpha \beta c}{a \beta+c-a \beta c}-1},-\infty<y<+\infty\right\}$ of $(0,0)$ in which the norms of all eigenvalues exceed 1 for all $(x, y) \in U$.

We can prove that in the neighborhood $U$, a point $z_{0}$ can be found such that the $M$ th iteration of $z_{0}$ under the map $F$ comes back to $(0,0)$ and $\operatorname{det}\left(D F\left(z_{k}\right)\right) \neq 0$ for $0 \leq k<M$, where $z_{k}=F^{k}\left(z_{0}\right)$. The detailed proof is given in the Appendix III.

Theorem 3. If $\left(a^{2} \beta^{2}-a \beta+1\right)\left(1+g^{2}\right)-\alpha \beta>0,4 \alpha \beta-\left(1+g^{2}\right)(a \beta-2)^{2}>0$, $a \beta>1$ and $2-a \beta-\frac{1}{a \beta}+\frac{\alpha}{a\left(1+g^{2}\right)} \neq 0$, then there exists at least a $c$ near $\frac{1}{a \beta}$ such that the origin is a snap-back repeller of map (1), and hence map (1) is chaotic in the sense of Marotto's definition.

\section{Numerical simulations.}

In this section, we present the bifurcation diagrams, the maximum lyapunov exponents corresponding to bifurcation diagram, phase portraits and iteration series for system (1) to demonstrate the above theoretical analysis and show the new interesting complex dynamical behaviors using numerical simulations.

Taking the values $a=0.8, \alpha=2.6, \beta=2.6, g=1$ in [15] and ranging $c$ from 0 to 1 , the map (1) becomes

$$
\left\{\begin{array}{l}
x \mapsto(1-c) x-2.6 c[0.8 y-k(x)], \\
y \mapsto y-2.6[0.8 y-k(x)]
\end{array}\right.
$$

where $k(x)=2.6[\arctan (x-1)-\arctan (-1)]$.

After simple calculation, one may discover that the fixed point $O$ of system (10) loses its stability at $c=c_{0}=0.0233918$ because of the flip bifurcation. And $a \beta=2.08>2,8+3 g-24 g^{2}+3 g^{3}=-10<0,4 \alpha \beta-\left(1+g^{2}\right)(a \beta-2)^{2}=$ $27.0272>0$. From Fig. 1(a) we can see that the period-2 points that bifurcate from this fixed point are stable. Moreover, system (10) generates an invariant circle(quasi-period orbit) while parameter $c$ goes through $c_{1}=0.466368$, which is the Naimark-Sacker bifurcation value. In fact, the Jacobian matrix of map (10) has a pair of complex conjugate eigenvalues:

$$
\lambda(c)=\frac{2.38 c-0.08+2.38 i \sqrt{(c-0.258162)(2.95856-c)}}{2},
$$




$$
\bar{\lambda}(c)=\frac{2.38 c-0.08-2.38 i \sqrt{(c-0.258162)(2.95856-c)}}{2}
$$

and it is easy to verify $|\lambda(0.466368)|=|\bar{\lambda}(0.466368)|=1, \lambda^{n} \neq 1, n=1,2,3,4$ and $\left.\frac{d|\lambda(c)|}{d c}\right|_{c=0.466368}=2.23 \neq 0$. And the value $\mathbf{a}=-0.0421979<0$, so there appears an attracting invariant circle.

Fig. 1(b) is the bifurcation diagram, showing the output of $x$ component with respect to the parameter $c$. The phase portrait of the circle for $c=0.467$ is shown in Fig. 3.

The maximum Lyapunov exponents are also calculated and plotted in Fig. 2 where we can easily see that the maximum Lyapunov exponents are negative for the parameter $c \in(0.1,0.467)$ while the origin is stable correspondingly. For $c \in(0.467,0.5)$ the maximum Lyapunov exponents are in the neighborhood of zero which is corresponding to quasi-period solutions. For $c \in(0.5,0.578)$, the maximum Lyapunov exponents are positive with a few are negative which shows that period window occurs in the chaotic region. In $(0.58,1)$, the maximum Lyapunov exponents are negative, there exist stable period points.

In addition, there are many complex dynamics: when $c$ range from 0.521 to 0.522 , the size of the attractor suddenly increases, that is to say interior crisis occurs(see Fig. 4(a),(b) $)^{[14,15]}$; for values of the parameter $c$ larger than the critical transition value $c_{T}$ (for example $c=0.5788$ ) the attractor is a periodic orbit, for $c$ slightly less than $c_{T}$ (for example $c=0.5787$ ) there long stretches of time during which the orbit appears to be periodic and closely resembles the orbit for $c>c_{T}$, but this regular(approximately periodic) behavior is intermittently interrupted by a finite duration 'burst' in which the orbit behaves in a decidedly different manner. This shows that the phenomenon of the Poneau-Manneville intermittency to a chaotic attractor occurs(see Fig. 4(c),(d)).

\section{Acknowledgement}

We would like to thank the reviewers and the editor for their careful reading of the original manuscript and many valuable comments and suggestions that greatly improved the presentation of this paper. 


\section{References}

[1] Chiarella, C., Dieci, R., Gardini, L. (2002). "Speculative behavior and complex asset price dynamics: a global analysis". Journal of Economic Behavior \& Organization, 49, 173-197.

[2] Fernandez-Rodriguez, F., Garcia-Artiles, M. D. and Martin Gonzalez, J. M. (2002). "A model of speculative bahaviour with a strange attractor". Applied Mathematical Finance, 9, 143-161.

[3] Gardini, L. (1994). "Homoclinic bifurcations in n-dimensional endomorphisms, due to expanding periodic points". Nonlinear Analysis: Theory, Method \& Applications, 23, 1039-1089.

[4] Guckenheimer, J., Holmes, P. (1997). Nonlinear oscillations, dynamical systems, and bifurcations of vector fields. Springer-Verlag, New York.

[5] Hirsch, M., Smale, S. (1974). Differential equations, dynamical systems, and linear algebra. Academic Press, New York.

[6] Huang, Y., Chen, G., Ma, D. W. (2006). "Rapid fluctuations of chaotic maps on $\mathrm{R}^{N}$ ". J. Math. Anal. Appl., 323(1), 228-252.

[7] Jiang, G. R., Lu, Q. S. (2006). "The dynamics of a prey-predator model with impulsive state feedback control". Discrete and Continuous Dynamical Systems-Series B, 6(6), 1301-1320.

[8] Jing, Z. J., Chang, Y., Guo, B. L. (2004). "Bifurcation and chaos in discrete FitzHugh-Nagumo system". Chaos Solit. Fract., 21, 701-720.

[9] Li, T. Y., Yorke, J. A. (1975). "Period three implies chaos". Amer. Math. Monthly, 82, 985-992.

[10] Lin, W., Ruan, J., Zhao, W. (2002). "On the mathematical clarification of the snap-back repeller in high-dimensional systems and chaos in a discrete neural network model". Int. J. Bifurcation and Chaos, 12(5), 1129-1139.

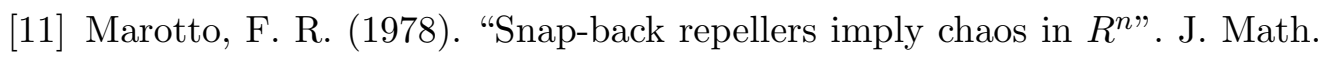
Anal. Appl., 63, 199-223. 
[12] Marotto, F. R. (2005). "On redefining a snap-back repeller". Chaos Solit. Fract., 25, 25-28.

[13] Medio, A., Lines, M. (2001). Nonlinear dynamics: a primer. Cambridge University Press, Cambridge.

[14] Ott, E. (1993). Chaos in dynamical systems. Cambridge University Press, Cambridge.

[15] Dieci, R. (2001). "Critical curves and bifurcations of absorbing areas in a financial model". Nonlinear Analysis: Theory, Method \& Applications, 47, 5265-5276.

[16] Robinson, C. (1999). Dynamical systems: stability, symbolic dynamics, and chaos. CRC Press, Second edition.

[17] Seydel, R. (1999). Practical bifurcation and stability analysis: from equilibrium to chaos. Springer-Verlag, Second edition.

[18] Wiggins, S. (1990). An introduction to applied nonlinear dynamics and chaos. Springer-Verlag, New York.

\section{Appendix I}

When $c=c_{0}$, one of the eigenvalues of $\operatorname{DF}(0,0)$ is $\lambda_{1}=-1$, the other is $\lambda_{2}=$ $-\frac{\left(1+g^{2}\right)(a \beta-1)(a \beta-2)-2 \alpha \beta}{\left(1+g^{2}\right)(a \beta-2)+2 \alpha \beta}$. And $\left|\lambda_{2}\right|<1$ if $4 \alpha \beta-\left(1+g^{2}\right)(a \beta-2)^{2}>0$.

Let $\tilde{c}=c-c_{0}$, we consider the parameter $\tilde{c}$ as a new and dependent variable, then map (1) becomes:

$$
\begin{aligned}
& \left(\begin{array}{l}
x \\
y \\
\tilde{c}
\end{array}\right) \mapsto\left(\begin{array}{ccc}
1-c_{0}+\frac{\alpha \beta c_{0}}{1+g^{2}}-a \beta c_{0} & 0 \\
\frac{\alpha \beta}{1+g^{2}} & 1-a \beta 0 \\
0 & 0 & 1
\end{array}\right)\left(\begin{array}{l}
x \\
y \\
\tilde{c}
\end{array}\right) \\
& +\left(\begin{array}{l}
-\tilde{c} x-a \beta \tilde{c} y+\beta \tilde{c} k(x)+c_{0} \beta\left[k(x)-\frac{\alpha}{1+g^{2}} x\right] \\
\beta\left[k(x)-\frac{\alpha}{1+g^{2}} x\right] \\
0
\end{array}\right) \\
& \text { Let } T=\left(\begin{array}{ccc}
1 & \frac{2 a \beta\left(1+g^{2}\right)}{\left(1+g^{2}\right)(a \beta-2)+2 \alpha \beta} & 0 \\
\frac{\alpha \beta}{\left(1+g^{2}\right)(a \beta-2)} & 1 & 0 \\
0 & 0 & 1
\end{array}\right) \text { and use the translation }
\end{aligned}
$$




$$
\begin{aligned}
& \left(\begin{array}{l}
x \\
y \\
\tilde{c}
\end{array}\right)=T\left(\begin{array}{l}
\tilde{x} \\
\tilde{y} \\
\mu
\end{array}\right) \text {, then map (2) becomes } \\
& \left(\begin{array}{c}
\tilde{x} \\
\tilde{y} \\
\mu
\end{array}\right) \mapsto\left(\begin{array}{ccc}
-1 & 0 & 0 \\
0 & -\frac{\left(1+g^{2}\right)(a \beta-1)(a \beta-2)-2 \alpha \beta}{\left(1+g^{2}\right)(a \beta-2)+2 \alpha \beta} & 0 \\
0 & 0 & 1
\end{array}\right)\left(\begin{array}{l}
\tilde{x} \\
\tilde{y} \\
\mu
\end{array}\right)+\left(\begin{array}{c}
f(\tilde{x}, \tilde{y}, \mu) \\
g(\tilde{x}, \tilde{y}, \mu) \\
0
\end{array}\right),
\end{aligned}
$$

where

$$
\begin{aligned}
f(\tilde{x}, \tilde{y}, \mu)= & -\frac{4 \alpha \beta g(a \beta-2)}{\left(1+g^{2}\right)\left[\left(1+g^{2}\right)(a \beta-2)^{2}-4 \alpha \beta\right]} \tilde{x}^{2} \\
& -\frac{16 \alpha a^{2} \beta^{3} g(a \beta-2)\left(1+g^{2}\right)}{\left[\left(1+g^{2}\right)(a \beta-2)+2 \alpha \beta\right]^{2}\left[\left(1+g^{2}\right)(a \beta-2)^{2}-4 \alpha \beta\right]} \tilde{y}^{2} \\
& -\frac{16 \alpha a \beta^{2} g(a \beta-2)}{\left[\left(1+g^{2}\right)(a \beta-2)+2 \alpha \beta\right]\left[\left(1+g^{2}\right)(a \beta-2)^{2}-4 \alpha \beta\right]} \tilde{x} \tilde{y} \\
& -\frac{\left[\left(1+g^{2}\right)(a \beta-2)+2 \alpha \beta\right]^{2}}{\left(1+g^{2}\left[\left(1+g^{2}\right)(a \beta-2)^{2}-4 \alpha \beta\right]\right.} \tilde{x} \mu-\frac{a^{2} \beta^{2}(a \beta-2)\left(1+g^{2}\right)}{\left(1+g^{2}\right)(a \beta-2)^{2}-4 \alpha \beta} \tilde{y} \mu \\
& +\mathcal{O}\left((|\tilde{x}|+|\tilde{y}|+|\mu|)^{3}\right), \\
g(\tilde{x}, \tilde{y}, \mu)= & \frac{\alpha \beta g(a \beta-2)^{2}}{\left(1+g^{2}\right)\left[\left(1+g^{2}\right)(a \beta-2)^{2}-4 \alpha \beta\right]} \tilde{x}^{2} \\
& +\frac{4 \alpha a^{2} \beta^{3} g(a \beta-2)\left(1+g^{2}\right)}{\left[\left(1+g^{2}\right)(a \beta-2)+2 \alpha \beta\right)^{2}\left[\left(1+g^{2}\right)(a \beta-2)^{2}-4 \alpha \beta\right]} \tilde{y}^{2} \\
& +\frac{4 \alpha a \beta^{2} g(a \beta-2)^{2}}{\left[\left(1+g^{2}\right)(a \beta-2)+2 \alpha \beta\right]\left[\left(1+g^{2}\right)(a \beta-2)^{2}-4 \alpha \beta\right]} \tilde{x} \tilde{y} \\
& +\frac{\alpha \beta\left[\left(1+g^{2}\right)(a \beta-2)+2 \alpha \beta\right]^{2}}{(a \beta-2)\left(1+g^{2}\right)^{2}\left[\left(1+g^{2}\right)(a \beta-2)^{2}-4 \alpha \beta\right]} \tilde{x} \mu+\frac{\alpha a^{2} \beta^{3}}{\left(1+g^{2}\right)(a \beta-2)^{2}-4 \alpha \beta} \tilde{y} \mu \\
& +\mathcal{O}\left((|\tilde{x}|+|\tilde{y}|+|\mu|)^{3}\right) .
\end{aligned}
$$

By center manifold theory, we know that the stability of $(\tilde{x}, \tilde{y})=(0,0)$ near $\mu=0$ can be determined by studying a one-parameter family of equations on a center manifold, which can be represented as follows $W^{c}(0)=\left\{(\tilde{x}, \tilde{y}, \mu) \in R^{3} \mid \tilde{y}=h(\tilde{x}, \mu), h(0,0)=0, D h(0,0)=0\right\}$, for $\tilde{x}$ and $\mu$ sufficiently small.

We assume a center manifold of the form

$$
h(\tilde{x}, \mu)=a_{1} \tilde{x}^{2}+a_{2} \mu \tilde{x}+a_{3} \mu^{2}+\mathcal{O}\left((|\tilde{x}|+|\mu|)^{3}\right) .
$$

The center manifold must satisfy

$$
\begin{aligned}
\mathcal{N}(h(\tilde{x}, \mu))= & h(-\tilde{x}+f(\tilde{x}, h(\tilde{x}, \mu), \mu), \mu)+\frac{\left(1+g^{2}\right)(a \beta-1)(a \beta-2)-2 \alpha \beta}{\left(1+g^{2}\right)(a \beta-2)+2 \alpha \beta} h(\tilde{x}, \mu) \\
& -g(\tilde{x}, h(\tilde{x}, \mu), \mu) \\
= & 0 .
\end{aligned}
$$

Equating terms of like powers to zero gives $a_{1}=\frac{\alpha g(a \beta-2)\left[\left(1+g^{2}\right)(a \beta-2)+2 \alpha \beta\right]}{a\left(1+g^{2}\right)^{2}\left[\left(1+g^{2}\right)(a \beta-2)^{2}-4 \alpha \beta\right]}, a_{2}=\frac{\alpha \beta\left[\left(1+g^{2}\right)(a \beta-2)+2 \alpha \beta\right]^{3}}{(a \beta-2)\left(1+g^{2}\right)^{2}\left[\left(1+g^{2}\right)(a \beta-2)^{2}-4 \alpha \beta\right]^{2}}, a_{3}=0$. 
The map restricted to the center manifold is given by

$$
\begin{aligned}
\tilde{x} \mapsto \tilde{f} & \tilde{x}, \mu)=-\tilde{x}-\frac{4 \alpha \beta g(a \beta-2)}{\left(1+g^{2}\right)\left[\left(1+g^{2}\right)(a \beta-2)^{2}-4 \alpha \beta\right]} \tilde{x}^{2}-\frac{\left[\left(1+g^{2}\right)(a \beta-2)+2 \alpha \beta\right]^{2}}{\left(1+g^{2}\right)\left[\left(1+g^{2}\right)(a \beta-2)^{2}-4 \alpha \beta\right]} \tilde{x} \mu \\
& -\frac{4}{3} \frac{\alpha \beta(a \beta-2)\left[4 a \beta\left(1+(3 \alpha \beta-2) g^{2}-3 g^{4}\right)+a^{2} \beta^{2}\left(3 g^{4}+2 g^{2}-1\right)+4\left(-1+2 g^{2}+3 g^{4}+\alpha\left(\beta-9 \beta g^{2}\right)\right)\right]}{\left(1+g^{2}\right)^{2}\left[\left(1+g^{2}\right)(a \beta-2)^{2}-4 \alpha \beta\right]^{2}} \tilde{x}^{3} \\
& +\mathcal{O}\left((|\tilde{x}|+|\mu|)^{3}\right) .
\end{aligned}
$$

Since

$$
\begin{aligned}
\alpha_{1}= & \left.\left(\frac{\partial \tilde{f}}{\partial \mu} \frac{\partial^{2} \tilde{f}}{\partial \tilde{x}^{2}}+2 \frac{\partial^{2} \tilde{f}}{\partial \tilde{x} \partial \mu}\right)\right|_{(0,0)}=-\frac{2\left[\left(1+g^{2}\right)(a \beta-2)+2 \alpha \beta\right]^{2}}{\left(1+g^{2}\right)\left[\left(1+g^{2}\right)(a \beta-2)^{2}-4 \alpha \beta\right]}, \\
\alpha_{2}= & \left.\left(\frac{1}{2}\left(\frac{\partial^{2} \tilde{f}}{\partial \tilde{x}^{2}}\right)^{2}+\frac{1}{3}\left(\frac{\partial^{3} \tilde{f}}{\partial \tilde{x}^{3}}\right)\right)\right|_{(0,0)} \\
= & -\left[8 \alpha \beta ( a \beta - 2 ) \left(16 \beta^{2}\left[-1+(8-3 a \beta) g^{2}+6(a \beta-2)^{2} g^{3}-3(a \beta-3) g^{4}\right] \alpha^{2}\right.\right. \\
& +4 \beta(a \beta-2)^{2}\left(1+g^{2}\right)^{2}\left[2+3(a \beta-4) g^{2}\right] \alpha+(a \beta-2)^{4}\left(1+g^{2}\right)^{2}\left(-1+2 g^{2}\right. \\
& \left.\left.\left.+3 g^{4}\right)\right)\right] /\left[3\left(1+g^{2}\right)^{3}\left[\left(1+g^{2}\right)(a \beta-2)^{2}-4 \alpha \beta\right]^{3}\right] \\
\triangleq & -\frac{8 \alpha \beta(a \beta-2) s(\alpha)}{3\left(1+g^{2}\right)^{3}\left[\left(1+g^{2}\right)(a \beta-2)^{2}-4 \alpha \beta\right]^{3}},
\end{aligned}
$$

where

$$
\begin{aligned}
s(\alpha)= & 16 \beta^{2}\left[-1+(8-3 a \beta) g^{2}+6(a \beta-2)^{2} g^{3}-3(a \beta-3) g^{4}\right] \alpha^{2} \\
& +4 \beta(a \beta-2)^{2}\left(1+g^{2}\right)^{2}\left[2+3(a \beta-4) g^{2}\right] \alpha+(a \beta-2)^{4}\left(1+g^{2}\right)^{2}\left(-1+2 g^{2}+3 g^{4}\right) \\
\triangleq & A_{1} \alpha^{2}+B_{1} \alpha+C_{1},
\end{aligned}
$$

then $B_{1}^{2}-4 A_{1} C_{1}=48 \beta^{2}(a \beta-2)^{6} g^{3}\left(1+g^{2}\right)^{3}\left[8+3 g-24 g^{2}+3 g^{3}\right]$.

Let $l(a)=-1+(8-3 a \beta) g^{2}+6(a \beta-2)^{2} g^{3}-3(a \beta-3) g^{4}$

$$
\begin{aligned}
& =6 \beta^{2} g^{3} a^{2}-3 \beta g^{2}\left(1+8 g+g^{2}\right) a+\left(9 g^{4}+24 g^{3}+8 g^{2}-1\right) \\
& \triangleq A_{2} a^{2}+B_{2} a+C_{2},
\end{aligned}
$$

then $B_{2}^{2}-4 A_{2} C_{2}=3 \beta^{2} g^{3}\left(1+g^{2}\right)\left[8+3 g-24 g^{2}+3 g^{3}\right]$.

If $8+3 g-24 g^{2}+3 g^{3}<0$, then $A_{1}=16 \beta^{2} l(a)>0$ and $B_{1}^{2}-4 A_{1} C_{1}<0$. Hence $8 \alpha \beta(a \beta-2) s(\alpha)>0$ for $a \beta>2$.

\section{Appendix II}

Let $T=\left(\begin{array}{c}\frac{a\left(1+g^{2}\right)\left[\left(1+g^{2}\right)(a \beta-2)+2 \alpha \beta\right]}{2 \alpha\left[\left(1+g^{2}\right)(a \beta-1)+\alpha \beta\right]}-\frac{a\left(1+g^{2}\right) \sqrt{1+g^{2}}\left[4 \alpha \beta-\left(1+g^{2}\right)(a \beta-2)^{2}\right]}{2 \alpha\left[\left(1+g^{2}\right)(a \beta-1)+\alpha \beta\right]} \\ 0\end{array}\right)$ and use the translation $\left(\begin{array}{l}x \\ y\end{array}\right)=T\left(\begin{array}{l}u \\ v\end{array}\right)$, map (1) becomes

$$
\begin{aligned}
\left(\begin{array}{l}
u \\
v
\end{array}\right) \mapsto & \left(\begin{array}{cc}
\frac{2 \alpha \beta-\left(1+g^{2}\right)\left(a^{2} \beta^{2}-2 a \beta+2\right)}{2\left[\left(1+g^{2}\right)(a \beta-1)+\alpha \beta\right]} & -\frac{a \beta \sqrt{\left(1+g^{2}\right)\left[4 \alpha \beta-\left(1+g^{2}\right)(a \beta-2)^{2}\right]}}{2\left[\left(1+g^{2}\right)(a \beta-1)+\alpha \beta\right]} \\
\frac{a \beta \sqrt{\left(1+g^{2}\right)\left[4 \alpha \beta-\left(1+g^{2}\right)(a \beta-2)^{2}\right]}}{2\left[\left(1+g^{2}\right)(a \beta-1)+\alpha \beta\right]} & \frac{2 \alpha \beta-\left(1+g^{2}\right)\left(a^{2} \beta^{2}-2 a \beta+2\right)}{2\left[\left(1+g^{2}\right)(a \beta-1)+\alpha \beta\right]}
\end{array}\right)\left(\begin{array}{l}
u \\
v
\end{array}\right) \\
& +\left(\begin{array}{c}
f(u, v) \\
g(u, v)
\end{array}\right)
\end{aligned}
$$

where

$$
f(u, v)=a_{u u} u^{2}+a_{u v} u v+a_{v v} v^{2}+a_{u u u} u^{3}+a_{u u v} u^{2} v+a_{u v v} u v^{2}+a_{v v v} v^{3}
$$




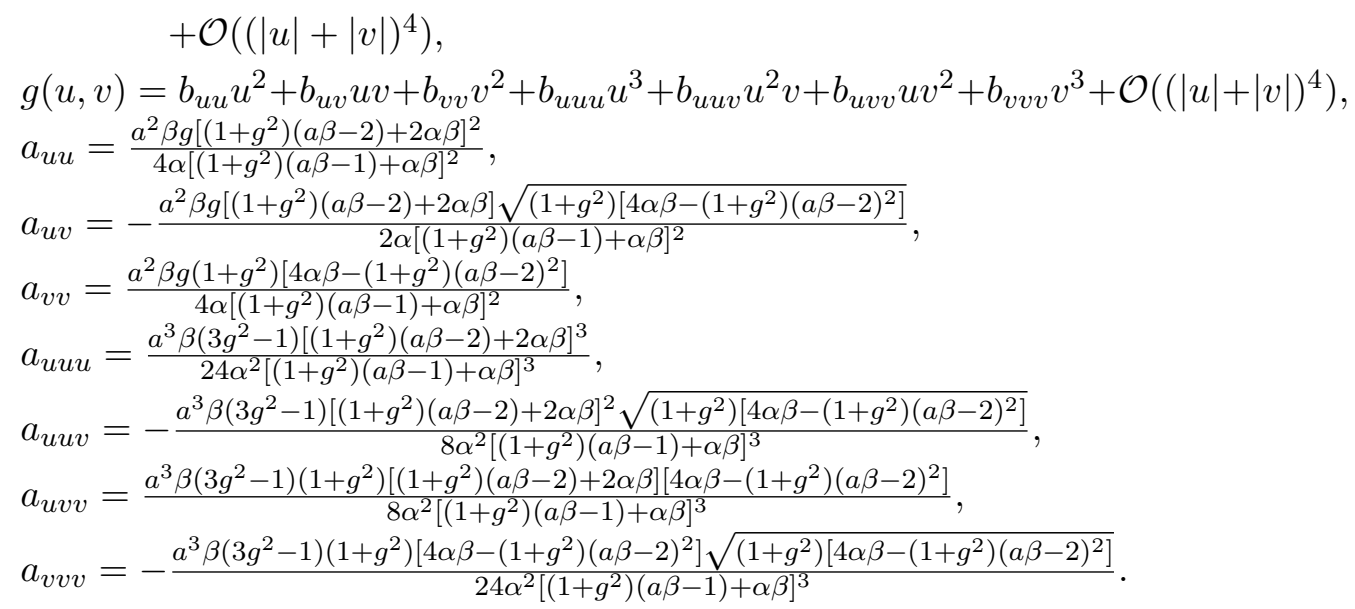

Notice that (6) is exactly in the form on the center manifold, in which the coefficient $\mathbf{a}$ is given by

$\mathbf{a}=-\operatorname{Re}\left[\frac{(1-2 \lambda) \bar{\lambda}^{2}}{1-\lambda} \xi_{11} \xi_{20}\right]-\frac{1}{2}\left|\xi_{11}\right|^{2}-\left|\xi_{02}\right|^{2}+\operatorname{Re}\left(\bar{\lambda} \xi_{21}\right)$,

where

$\xi_{20}=\frac{1}{8}\left[\left(f_{u u}-f_{v v}+2 g_{u v}\right)+i\left(g_{u u}-g_{v v}-2 f_{u v}\right)\right]$,

$\xi_{11}=\frac{1}{4}\left[\left(f_{u u}+f_{v v}\right)+i\left(g_{u u}+g_{v v}\right)\right]$,

$\xi_{02}=\frac{1}{8}\left[\left(f_{u u}-f_{v v}-2 g_{u v}\right)+i\left(g_{u u}-g_{v v}+2 f_{u v}\right)\right]$,

and

$\xi_{21}=\frac{1}{16}\left[\left(f_{u u u}+f_{u v v}+g_{u u v}+g_{v v v}\right)+i\left(g_{u u u}+g_{u v v}-f_{u u v}-f_{v v v}\right)\right]$.

Thus, an complex calculation gives

$\mathbf{a}=\frac{a^{3} \beta^{3}}{16\left[\left(1+g^{2}\right)(a \beta-1)+\alpha \beta\right]^{6}}\left(e_{1}-e_{2}+e_{3}+e_{4}-e_{5}+\frac{e_{6}}{4 \alpha \beta-\left(1+g^{2}\right)(a \beta-2)^{2}}\right)$,

where

$$
\begin{aligned}
e_{1}= & 2\left(3 g^{2}-1\right)\left[\left(1+g^{2}\right)(a \beta-1)+\alpha \beta\right]^{4}, \\
e_{2}= & \frac{12 a \alpha \beta^{2} g^{2}\left[\left(1+g^{2}\right)(a \beta-1)+\alpha \beta\right]^{4}}{4 \alpha \beta-\left(1+g^{2}\right)(a \beta-2)^{2}}, \\
e_{3}= & a^{2} \beta^{2} g^{2}(a \beta-2)\left[\alpha \beta-\left(1+g^{2}\right)\right]\left(1+g^{2}\right)\left[a^{4} \beta^{4}\left(1+g^{2}\right)^{2}-a^{3} \beta^{3}\left(1+g^{2}\right)^{2}\right. \\
& \left.-a^{2} \alpha \beta^{3}\left(1+g^{2}\right)-2 a \beta\left[\alpha \beta-\left(1+g^{2}\right)\right]\left(1+g^{2}\right)-\left(1+g^{2}-\alpha \beta\right)^{2}\right], \\
e_{4}= & a^{2} \beta^{2} g^{2}\left(1+g^{2}\right)\left[2 \alpha \beta+\alpha^{2} \beta^{2}-2\left(1+g^{2}\right)+a \beta\left(1+g^{2}\right)\right] \\
& {\left[a^{4} \beta^{4}\left(1+g^{2}\right)^{2}-a^{3} \beta^{3}\left(1+g^{2}\right)^{2}-a^{2} \alpha \beta^{3}\left(1+g^{2}\right)\right.} \\
& \left.-2 a \beta\left[\alpha \beta-\left(1+g^{2}\right)\right]\left(1+g^{2}\right)-\left(1+g^{2}-\alpha \beta\right)^{2}\right], \\
e_{5}= & a^{3} \beta^{3} g^{2}\left[\alpha \beta-\left(1+g^{2}\right)\right]\left(1+g^{2}\right)\left[a^{4} \beta^{4}\left(1+g^{2}\right)^{2}+a^{2} \beta^{2}\left(1+g^{2}\right)\left[2\left(1+g^{2}\right)-3 \alpha \beta\right]\right. \\
& \left.-2 a \beta\left[\alpha \beta-\left(1+g^{2}\right)\right]\left(1+g^{2}\right)-3 a^{3} \beta^{3}\left(1+g^{2}\right)^{2}-\left(1+g^{2}-\alpha \beta\right)^{2}\right], \\
e_{6}= & a^{3} \beta^{3} g^{2}(a \beta-2)\left(1+g^{2}\right)^{2}\left[\alpha \beta(\alpha \beta+2)+\left(1+g^{2}\right)(a \beta-2)\right] \\
& {\left[a^{4} \beta^{4}\left(1+g^{2}\right)^{2}+a^{2} \beta^{2}\left(1+g^{2}\right)\left[2\left(1+g^{2}\right)-3 \alpha \beta\right]-2 a \beta\left[\alpha \beta-\left(1+g^{2}\right)\right]\left(1+g^{2}\right)\right.}
\end{aligned}
$$




$$
\left.-3 a^{3} \beta^{3}\left(1+g^{2}\right)^{2}-\left(1+g^{2}-\alpha \beta\right)^{2}\right] .
$$

\section{Appendix III}

In fact we have

$$
\begin{aligned}
& \left\{\begin{array}{c}
(1-c) x-c \beta[a y-k(x)]=x^{*}, \\
y-\beta[a y-k(x)]=y^{*},
\end{array}\right. \\
& \left\{\begin{array}{cc}
(1-c) x-c \beta[a y-k(x)] & =0, \\
y-\beta[a y-k(x)] & =0 .
\end{array}\right.
\end{aligned}
$$

Now a $F^{2}$ map has been constructed to map the point $z_{0}$ to the fixed point $O$ after two iterations when there are nonzero solutions for Equations (8) and (9). Through straightforward calculation, the nonzero solution for (9) should satisfy the following equation:

$$
\left\{\begin{array}{l}
(1-c)(1-a \beta) x+c \beta k(x)=0 \\
y=\frac{1-c}{c} x
\end{array}\right.
$$

Let $f(x)=(1-c)(1-a \beta) x+c \beta k(x)$. When $g-\sqrt{\frac{\alpha \beta c}{(a \beta-1)(1-c)}-1}<x<$ $g+\sqrt{\frac{\alpha \beta c}{(a \beta-1)(1-c)}-1}\left(c>\frac{a \beta-1}{a \beta-1+\alpha \beta}, a \beta>1\right)$ we have $f^{\prime}(x)>0$. And when $c>c_{1}$ we have $f^{\prime}(0)>0$. Because $\lim _{x \rightarrow+\infty} f(x)=-\infty$, there exists $x^{*}=x^{*}(c)>$ $g+\sqrt{\frac{\alpha \beta c}{(a \beta-1)(1-c)}-1}$ such that $f\left(x^{*}\right)=0, y^{*}=\frac{1-c}{c} x^{*} . x^{*}$ is continuous with respect to $c$.

The nonzero solution for (8) should satisfy the following equation:

$$
\left\{\begin{array}{l}
(1-c)(1-a \beta) x+c \beta k(x)+(a \beta c-1) x^{*}=0, \\
y=\frac{1-c}{c} x-x^{*}
\end{array}\right.
$$

Let $\phi(c, x)=(1-c)(1-a \beta) x+c \beta k(x)+(a \beta c-1) x^{*}$, then

(1) $\phi\left(\frac{1}{a \beta}, 0\right)=0$,

$(2) \phi(c, x)$ is continuous on $[0,1] \times R$,

(3) $\frac{\partial \phi}{\partial x}\left(\frac{1}{a \beta}, 0\right)=2-a \beta-\frac{1}{a \beta}+\frac{\alpha}{a\left(1+g^{2}\right)}$,

if we require that $2-a \beta-\frac{1}{a \beta}+\frac{\alpha}{a\left(1+g^{2}\right)} \neq 0$, then there exist $x=x_{0}(c)$ and $\rho>0$ such that

(i) $\phi\left(c, x_{0}(c)\right)=0$ for $c \in\left(\frac{1}{a \beta}-\rho, \frac{1}{a \beta}+\rho\right)$ such that $x_{0}\left(\frac{1}{a \beta}\right)=0$,

(ii) $x=x_{0}(c)$ is continuous in $\left(\frac{1}{a \beta}-\rho, \frac{1}{a \beta}+\rho\right)$.

Let $M=2, z_{0}=\left(x_{0}, y_{0}\right)=\left(x_{0}, \frac{1-c}{c} x_{0}-x^{*}\right)$, then $z_{0}$ locates in $U$ for $c-\frac{1}{a \beta}$ small 
enough.

The condition $\frac{1}{a \beta}>c_{1}$ leads to $\left(a^{2} \beta^{2}-a \beta+1\right)\left(1+g^{2}\right)-\alpha \beta>0$, and $\frac{1}{a \beta}<1$ requires $a \beta>1$.

Through simple calculation, we know that only if $x=g \pm \sqrt{\frac{\alpha \beta c}{(a \beta-1)(1-c)}-1}, D F(x, y)=$ 0. So, $D F\left(z_{0}\right) \neq 0, D F\left(F\left(z_{0}\right)\right) \neq 0$.

Let $U^{*}=\left\{(x, y)\left|\frac{x^{2}}{r_{x_{0}}^{2}}+\frac{y^{2}}{r_{y_{0}}^{2}} \leq 1,\right| x_{0}\left|<r_{x_{0}}, r_{y_{0}}=\right| y_{0} \mid+\eta, \eta\right.$ is some positive constant $\}$, where $r_{x_{0}}<\min \left\{\left|g-\sqrt{\frac{\alpha \beta c}{a \beta+c-a \beta c}-1}\right|, g+\sqrt{\frac{\alpha \beta c}{a \beta+c-a \beta c}-1}\right\}$. Then $z_{0}$ is a snapback repeller in $U^{*}$. 


\section{Appendix IV}

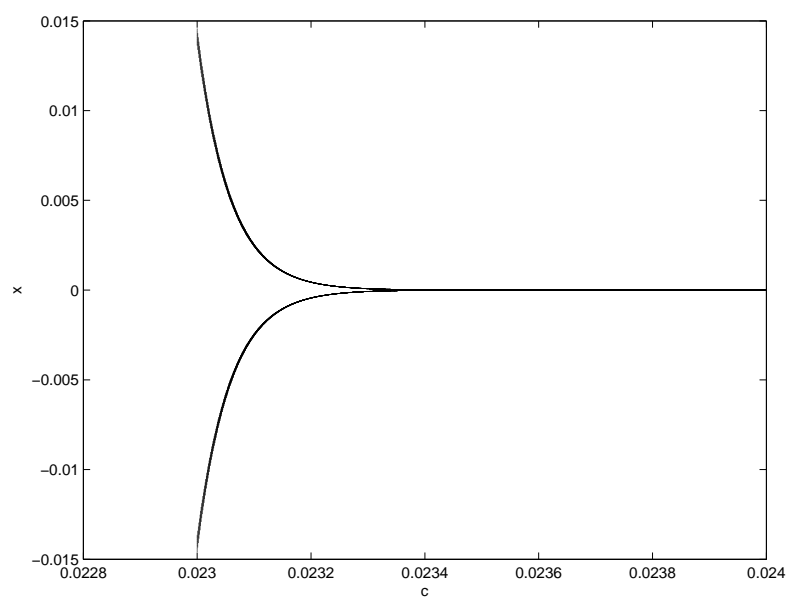

(a)

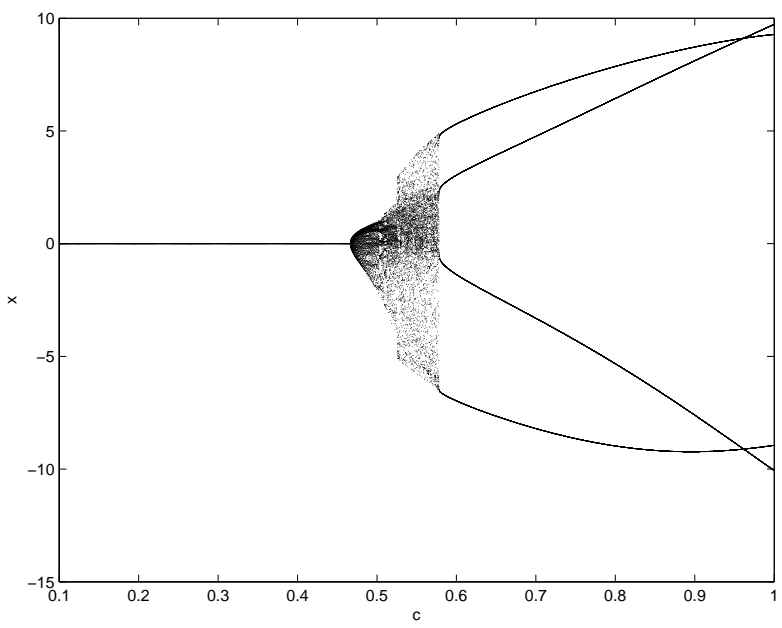

(b)

Fig. 1 Bifurcation diagram of $x$ output vs $c$ where the initial values are $x_{0}=$ $0.001, y_{0}=0.001$. 


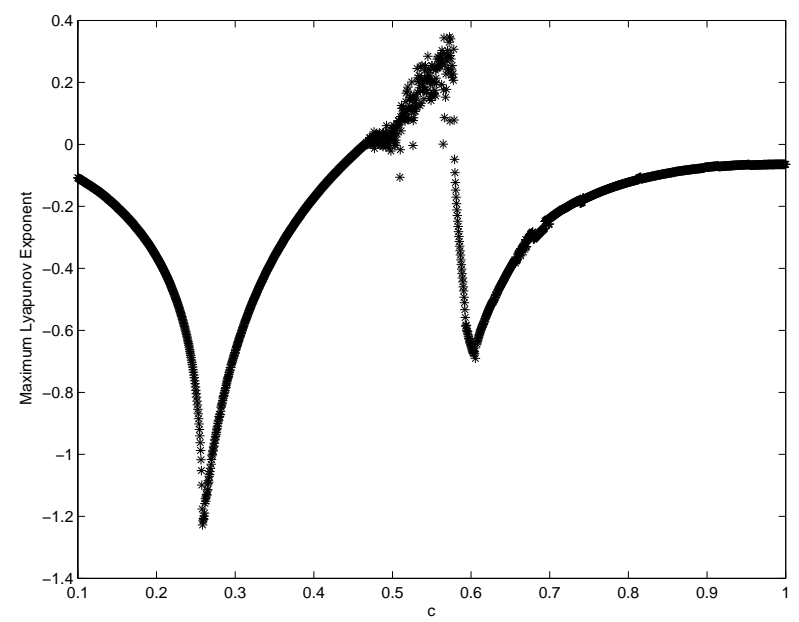

Fig. 2 The maximum Lyapunov exponent vs the parameter $c$. The initial values are $x_{0}=0.001, y_{0}=0.001$.

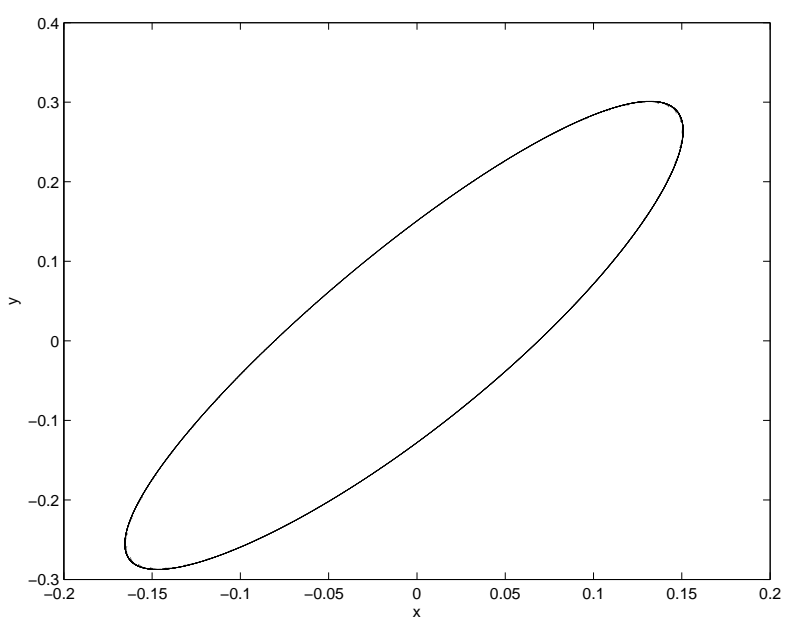

Fig. 3 Phase portrait of map (10) for $c=0.467$. 


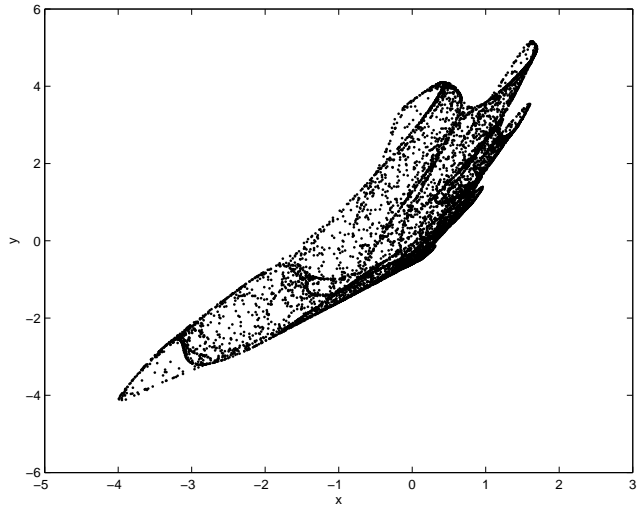

(a) $c=0.521$

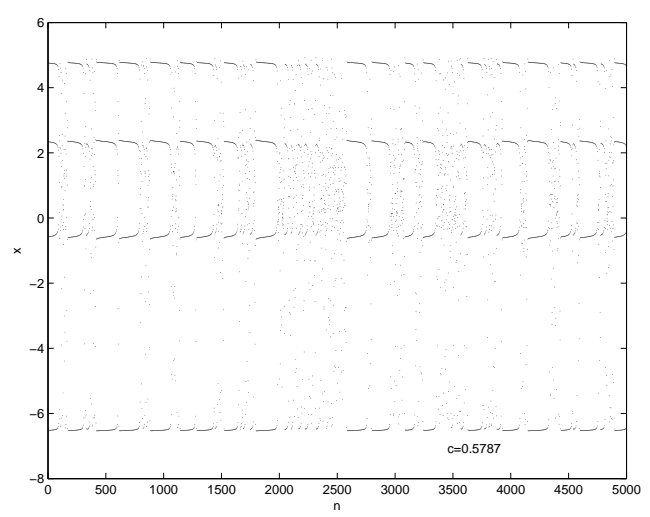

(c) $c=0.5787$

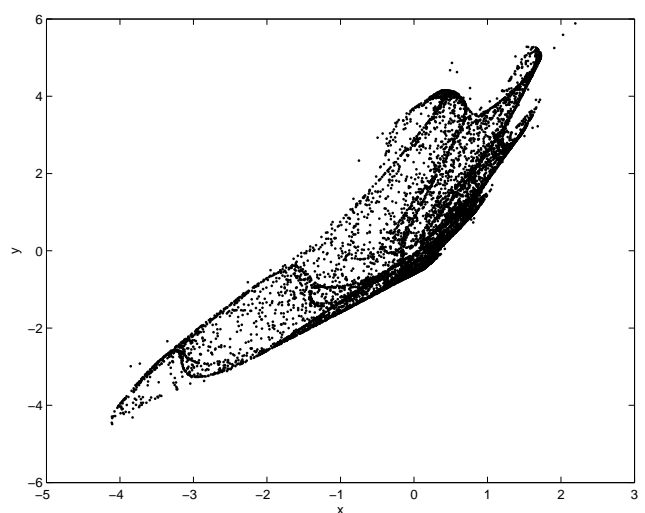

(b)c $=0.522$

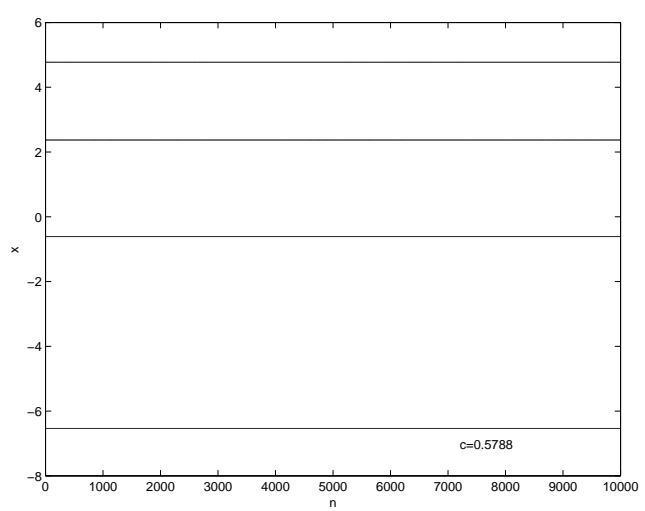

$(d) c=0.5788$

Fig. 4 Interior crisis and Poneau-Manneville intermittency. 
Yinghui Gao

School of Mathematics and System Sciences

Beihang University

LMIB of the Ministry of Education

Beijing 100191

P. R. China

Email: yhgao@buaa.edu.cn

Wei Feng

School of Mathematics and System Sciences

Beihang University

LMIB of the Ministry of Education

Beijing 100191

P. R. China

Bing Liu

Department of Mathematics

Anshan Normal University

Anshan, 114005, Liaoning

P. R. China. 